\title{
Cutaneous Adverse Reactions to Highly Antiretroviral Therapy in HIV-Positive Patients
}

\author{
G. Pistone A. Pistone D. Sorbello E. Viviano M.R. Bongiorno \\ Department of Dermatology, University of Palermo, Palermo, Italy
}

\section{Key Words}

HIV · Adverse drug reactions · Skin · Highly antiretroviral therapy

\begin{abstract}
Adverse drug reactions to highly antiretroviral therapy (HAART) are major obstacles in its success. Although overall mortality from HIV has dramatically declined owing to HAART, these antiretroviral regimens have been associated with a wide spectrum of severe cutaneous reactions. The severity of cutaneous adverse reactions varies greatly, and some may be difficult to manage. To optimize adherence and efficacy of antiretroviral treatment, clinicians must focus on preventing adverse effects whenever possible, and distinguish those that are self-limited from those that are potentially serious. This paper presents the case of a serious cutaneous adverse reaction to Atripla in a HIV-positive 50-year-old Caucasian woman.
\end{abstract}

(C) 2014 S. Karger AG, Basel

\section{Case Report}

In the present report, we describe the case of a serious cutaneous adverse reaction that arose in a HIV-positive 50-year-old Caucasian woman. The patient, who had been HIV positive for about 2 years, was naive to all antiretroviral agents. She was asymptomatic. In June 2010, antiretroviral therapy with Atripla [1] was started, because of a rapid decrease in her CD4+ T cell counts and a significant increase of HIV RNA viral load. She had a virologic response and an increase in CD4 cell count within 1 month of therapy. Therefore, the initial symptoms of the cutaneous reaction started during the 3 weeks after the beginning of Atripla treatment. The cutaneous eruption started as a macular erythema that evolved into a red, symmetrical, confluent, papular eruption. Initially, the upper trunk, face, and pressurebearing areas were affected with a later symmetrical involvement of the extremities (fig. 1,

Maria Rita Bongiorno, MD

Department of Dermatology, University of Palermo

Via del Vespro 131

IT-90127 Palermo (Italy)

E-Mail mariarita.bongiorno@unipa.it 
Pistone et al.: Cutaneous Adverse Reactions to Highly Antiretroviral Therapy in HIVPositive Patients

fig. 2). The cutaneous reaction was promptly treated with oral administration of prednisone at a dosage of $25 \mathrm{mg} /$ day, without withdrawing the antiretroviral medication. In spite of that, after 1 week, the reaction started to worsen and the patient was referred to our institution for the appearance of large erythematous and edematous plaques that might range from red to flesh-colored to yellow and covered large areas of the body. The lesions progressed and soon merged together, resulting in a widespread erythematous rash tending to erythroderma with pityriasis-like scales, and the skin was universally involved, from head to foot. The patient complained about a feeling of sickness. Facial and periorbital edema were easily recognized and led to gross distortion of the patient's features, associated with a feeling of tension. Mucosal involvement was not present. The development of these skin manifestations was associated with fever, severe malaise, chills, and pruritus [2-4]. Fever was high and spiking, racing from 38 to $39^{\circ} \mathrm{C}$. The dermopathy was quite severe and required hospitalization. At admission, the physical examination of the patient was unremarkable. A routine blood test revealed raised inflammatory markers, C-reactive protein was $3.5 \mathrm{mg} / \mathrm{dl}$, and erythrocyte sedimentation rate was $24 / \mathrm{h}$, and there was lymphopenia ( 580 cells/ $\mu$ l). Liver and renal function tests were normal; no eosinophilia was present. CD4+ T cell counts and HIV RNA viral load were 341 cells $/ \mu \mathrm{l}$ and 39,000 copies $/ \mathrm{ml}$, respectively. A chest X-ray did not show significant alterations. The spikes in temperature generated concern for a suspected underlying infection, but results of various cultures were negative, a finding that is especially significant in a patient with a severe reaction. The patient had not taken any drugs except the antiretroviral agents in the month preceding the appearance of the cutaneous eruption. Therefore, the antiretroviral treatment was discontinued and a therapy with oral corticosteroids (50 mg prednisone equivalent daily) and antihistaminic drugs (10 mg cetirizine dichloride equivalent daily) was promptly administered with simultaneous administration of antacids to prevent peptic ulcers. They had marked anti-exudative, anti-inflammatory, and anti-allergic effects, and there was a significant improvement within a few days. Consequently, they suppressed the intensity of the reaction, controlled the extension of the skin eruption, decreased the involved area, reduced fever, which disappeared within 4 days, and discomfort. The therapy was very effective and shortened the course of the dermopathy. However, clinical improvement of the patient declined gradually. This dosage of oral therapy was maintained for 3 weeks until progression of the skin lesions halted and there was complete clinical remission. Subsequently, the dose of prednisone was reduced gradually to a $5 \mathrm{mg}$ prednisone equivalent every week [5]. The patient remained asymptomatic. Three months after the corticosteroid was stopped, she remained free of cutaneous lesions.

\section{Discussion}

The arrival of the new drug Atripla has been a noteworthy milestone in the treatment of HIV infection, simplifying administration with the intent of increasing adherence and because successful management of HIV infection requires the simultaneous use of 3 or more drugs [6-8]. This approach has significantly simplified the handing of highly antiretroviral therapy (HAART), thus improving patients' compliance and the subsequent clinical outcome for its excellent potency, tolerability, and favorable safety profile $[9,10]$. On the other hand, Atripla has not decreased the rate of systemic and cutaneous adverse reactions in HIV patients. Fortunately, although adverse cutaneous reactions to Atripla occur frequently, only a small proportion of them are severe and very few are fatal. Most of the skin reactions are of minor relevance and do not require the withdrawal of the antiretroviral treatment, being 
Pistone et al.: Cutaneous Adverse Reactions to Highly Antiretroviral Therapy in HIV Positive Patients

cured with low doses of corticosteroids; however, particularly in severe cases, an interruption of the therapy is mandatory. This was done in our case because of the fever and incipient erythroderma. In fact, it is well known that maculopapular drug eruptions can occasionally evolve into exfoliative erythroderma [11], although it is not yet clear whether they can progress into Stevens-Johnson syndrome or toxic epidermal necrolysis [12]. In addition, since each of the three drugs alone can cause skin reactions, it was impossible for us to identify which of the preparations contained in the tablet had been responsible for the cutaneous eruption. Patients infected with HIV have a higher risk of developing cutaneous reactions to specific medications compared with the general population. This is due to the large number of medicaments to which they are exposed and presumably a result of their TH2/TH1 lymphocyte imbalance and altered drug metabolism [13]. Consequently, adverse reactions, and not treatment failure, represent the most common reasons for discontinuing HAART within the first year of therapy [14]. Therefore, knowledge of adverse reactions to antiretroviral drugs is necessary as is the reporting of new ones, especially taking into account the possibility of severe and sometimes life-threatening reactions. Dermatologists play an important role in the management of these cutaneous effects of HAART treatment in HIV patients and they should be familiar with these conditions and be prepared to handle them adequately. The rapid detection and treatment of cutaneous adverse drug reactions, plus the identification of the causative agents, are essential for preventing a progression of the reaction. It is necessary not only to prevent additional exposures but also to ensure the appropriate use of medications according to the patient's clinical parameters such as age. Prompt recognition and diagnosis and early identification and withdrawal of all potential causative drugs are, for the time being, the best we can do for our patients to most significantly influence outcome and prognosis [15]. As suggested by many authors, future studies of antiretroviral medications should have longer follow-ups to seek further evidence on recurrence risk, long-term safety profile, dosing regimens, and suitable management of the potential side effects in addition to the benefits of HAART.

\section{Disclosure Statement}

The authors have no conflicts of interest to disclose.

\section{References}

1 Yeni P: Update on HAART in HIV. J Hepatol 2006;44:S100-S103.

-2 Gonzales-Martin G, Yanez CG, Gonzales-Contreras L, et al: Adverse drug reactions in patients with HIV infection. A prospective study. Int J Clin Pharmacol Ther 1999;37:34-40.

-3 Borras-Blasco J, Navarro-Ruiz A, et al: Adverse cutaneous reactions associated with the newest antiretroviral drugs in patients with human immunodeficiency virus infection. J Antimicrob Chemother 2008;62:879-888.

4 Khalili H, Dashti-Khavidaki S, Mohraz M, et al: Antiretroviral induced adverse drug reactions in Iranian human immunodeficiency virus positive patients. Pharmacoepidemiol Drug Saf 2009;18:848-857.

5 Rudorf DC, Krikorian SA: Adverse effects associated with antiretroviral therapy and potential management strategies. J Pharm Pract 2005;18:258-277.

6 Goicoechea M, Best B: Efavirenz/emtricitabine/tenofovir disoproxil fumarate fixed-dose combination: first line therapy for all? Expert Opin Pharmcother 2007;8:371-382.

7 Long MC, King JR, Acosta EP: Pharmacologic aspects of new antiretroviral drugs. Curr Infect Dis Rep 2008;10:522-529.

-8 Feng JY, Ly JK, Myrick F, et al: The triple combination of tenofovir, emtricitabine and efavirenz shows synergistic anti-HIV-1 activity in vitro: a mechanism of action study. Retrovirology 2009;6:44. 
Pistone et al:: Cutaneous Adverse Reactions to Highly Antiretroviral Therapy in HIVPositive Patients

9 Knobel H, Carmona A, Grau S, et al: Adherence and effectiveness of highly active antiretroviral therapy. Arch Int Med 1998;158:1953.

10 Paterson DL, Swindells S, Mohr J, et al: Adherence to protease inhibitor therapy and outcomes in patients with HIV infection. Ann Intern Med 2000;133:21-30.

11 Revuz J, Valeyrie-Allanore L: Drug reactions; in Bolognia JL, Jorrizzo JL, Rapini RP (eds): Dermatology. London, Mosby, 2003, pp 333-353.

12 O'Brien ME, Clark RA, Besch CL, Myers L, Kissinger P: Patterns and correlates of discontinuation of the initial HAART regimen in an urban outpatient cohort. J Acquir Immune Defic Syndr 2003;34:407-414.

-13 Manzardo C, Zaccarelli M, Aguero F, et al: Optimal timing and best antiretroviral regimen in treatment-naive HIV-infected individuals with advance diseases. J Acquir Immune Defic Syndr 2007;46(suppl 1):S9-S18.

14 Breathnach SM, Hinter H: Adverse Drug Reactions and the Skin. Oxford, Blackwell Scientific Publications, 1992.

15 Wolf R, Orion E, et al: Life-threatening acute adverse cutaneous drug reactions. Clinics Dermatol 2005;23:171-181.
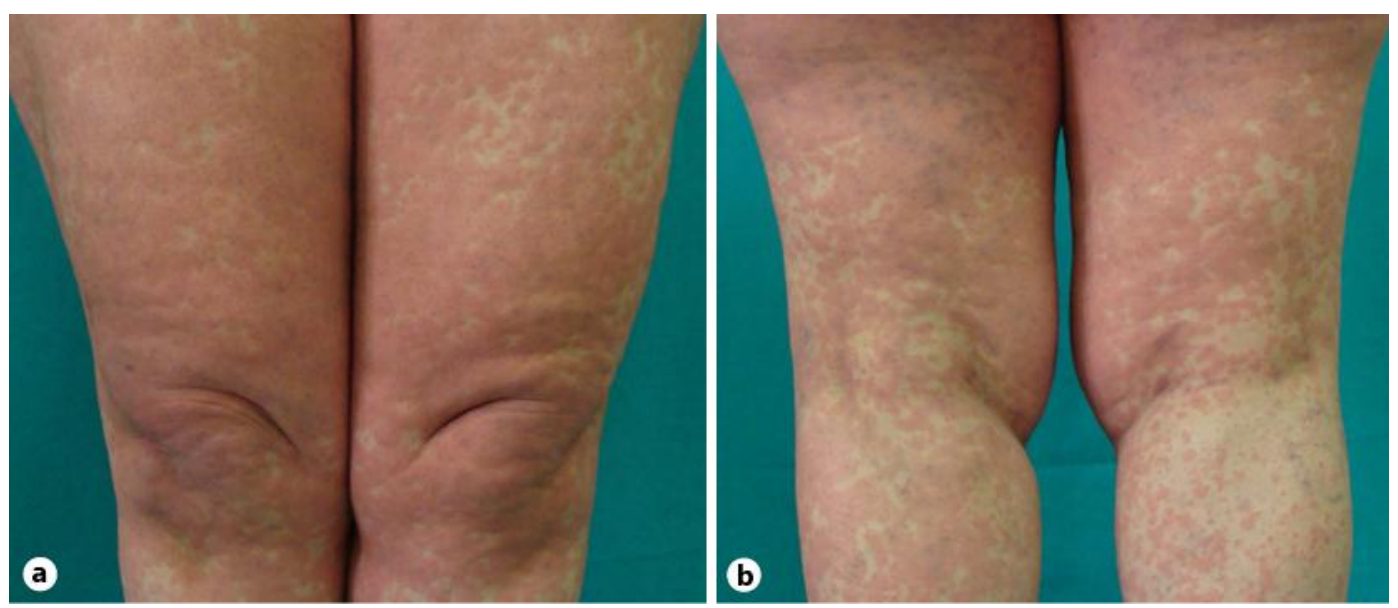

Fig. 1. Macular erythema with papular eruption in the upper trunk. 


\section{Case Reports in Dermatology}

Case Rep Dermatol 2014;6:145-149

DOI: $10.1159 / 000354030$

Pistone et al:: Cutaneous Adverse Reactions to Highly Antiretroviral Therapy in HIVPositive Patients
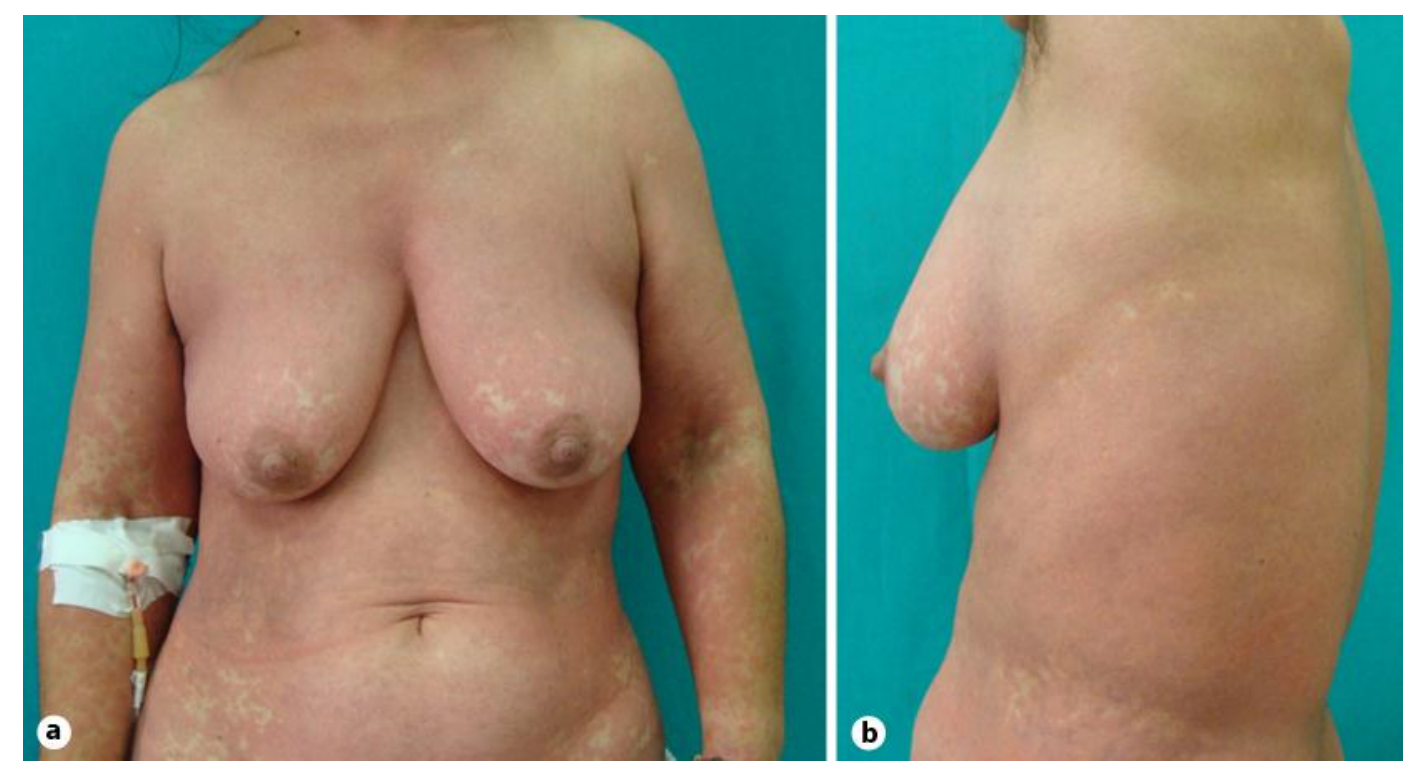

Fig. 2. Symmetrical involvement of the extremities. 